Article

\title{
On algebraic properties of fundamental group of intuitionistic fuzzy topological spaces (IFTSs)
}

\author{
Laaro Abdullateef \\ Department of Mathematical Sciences, Bayero University Kano, Kano, Nigeria.; laaroabdullateef@gmail.com
}

Received: 28 August 2019; Accepted: 17 February 2019; Published: 7 March 2020.

\begin{abstract}
In this paper, the notion of some algebraic properties of fundamental group of intuitionistic fuzzy topological spaces (IFTSs) are introduced. We give a necessary and sufficient condition for a fundamental group of IFTSs to be abelian, a necessary and sufficient conditions for a subset of fundamental group of IFTSs to be subgroup, a necessary and sufficient condition for a subgroup of fundamental group of IFTSs to be normal and a necessary and sufficient condition for an element to be in a center of fundamental group of IFTSs. We also describe the set of centralizers of an element in a fundamental group of IFTSs and the quotient fundamental group of IFTSs.
\end{abstract}

Keywords: Intuitionistic fuzzy sets, intuitionistic fuzzy topology, fundamental group of IFTSs, abelian fundamental group, normal subgroup, quotient group.

MSC: 20N05, 54A40, 57M05.

\section{Introduction}

$\mathbf{T}$ he concept of intuitionistic fuzzy set was introduced as a tool for dealing with uncertainties. It was first defined by Atanassove [1] as generalization of fuzzy set introduced by Zadeh [2]. After the work of Atanassove [1], many researcher worked in this direction, for example, Az-zo'bi et al. [3] defined and studied the fundamental group of intuitionistic fuzzy topological spaces which depends on the concepts of intuitionistic fuzzy sets and intuitionistic fuzzy topology. This concept was completely described as a generalization of the fundamental group of fuzzy topological spaces. Osmanglu and Tokat [4] discussed the intuitionistic fuzzy soft topology and Babitha and John [5] generalized intuitionistic fuzzy soft sets and set theoretical operations with illustrating examples.

Soroja and Kalaichelvi [6] construct a topology on an intuitionistic fuzzy neutrosophic soft sets. The concepts of intuitionistic fuzzy neutrosophic soft closure, intuitionistic fuzzy neutrosophic soft interior, intuitionistic fuzzy neutrosophic soft exterior, intuitionistic fuzzy neutrosophic soft boundary were introduced and some of its properties were also studied. Deschrijver et al. [7] introduced the notion of intuitionistic fuzzy t-norm and t-conorm, and investigate under which conditions a similar representation theorem can be obtained. They also argued in [8] that some of the existing definitions that appear in intuitionistic fuzzy literature (i. e., intuitionistic fuzzy connectives: negation, conjunction, disjunction and implication) were not sufficiently general for all pratical purposes, and suggest to replace them with new ones.

Liu and Wang [9] introduced new methods for solving multi-criteria decision-making problem in an intuitionistic fuzzy environment. Ersoy et al. [10] introduced the concept of intuitionistic fuzzy soft rings, and some basic properties of intuitionistic fuzzy soft rings were also given.

Jiang [11] studied a new entropy and its properties based on the improved axiomatic definition of intuitionistic fuzzy entropy and the nonlinear triangular intuitionistic fuzzy number and its application in linear integral equation was proposed by Mondal et al. [12]. And also in the pipeline of the literature Li and Jin [13] proposed a scalar expected value of intuitionistic fuzzy random individuals and its application to risk evaluation in insurance companies.

As described by Az-zo'bi et al. [3], the operation on the fundamental group of intuitionistic fuzzy topological spaces is not trivial, hence the study of its algebraic properties is very essential, which is the main task of this paper. 


\section{Preliminaries}

Here we give some basic definitions and known results, which will help in proving main results of this paper.

Definition 1. [14] Let $X$ be a set and $I$ be a unit interval [0,1]. A fuzzy set $X$ is characterized by a membership function $\mu_{A}$ which associates with each point $x \in X$ its "grade of membership" $\mu_{A}(x) \in I$.

Definition 2. [15] An intuitionistic set $A$ is an object having the form $\left\langle X, A_{1}, A_{2}\right\rangle$, where $A_{1}$ and $A_{2}$ are subsets of $X$ satisfying $A_{1} \cap A_{2}=\varnothing$. The set $A_{1}$ is called the set of members of $A$, while $A_{2}$ is called the set of nonmembers of $A$.

Definition 3. [16] Let $X$ be nonempty fixed set. An intuitionistic fuzzy set $A$ (IFS for short) in the universe of discourse $\mathrm{X}$ is an object having the form $A=\left\{\left\langle x, \mu_{A}(x), v_{A}(x)\right\rangle: x \in X\right\}$, where the functions $\mu_{A}: X \rightarrow I$ and $v_{A}: X \rightarrow I$ denotes the degree of membership (namely $\mu_{A}(x)$ ) and degree of non-membership (namely $\left.\mu_{A}(x)\right)$ of each element $\mathrm{x} \in \mathrm{U}$ to the set $\mathrm{A}$, respectively, and $0 \leq \mu_{A}(x)+v_{A}(x) \leq 1$ for all $\mathrm{x} \in \mathrm{X}$.

Every fuzzy set $\mathrm{A}$ on a nonempty set $\mathrm{X}$ is obviously an IFS having the form $A=\left\{\left\langle x, \mu_{A}(x), 1-\mu_{A}(x)\right\rangle\right.$ : $x \in X\}$.

Definition 4. [17] Let $\mathrm{X}$ be nonempty set and $\mathrm{c} \in \mathrm{X}$ be a fixed element in $\mathrm{X}$. If $\lambda \in(0,1]$ and $\delta \in[0,1)$ are two fixed real numbers such that $\lambda+\delta \leq 1$, then the IFS

$$
c(\lambda, \delta)=\left\langle x, c_{\lambda}, 1-c_{1-\delta}\right\rangle
$$

is called intuitionistic fuzzy point (IFP) in X. If $\delta \in[0,1)$ is a fixed real number, then the IFS

$$
c(\delta)=\left\langle x, 0,1-c_{1-\delta}\right\rangle
$$

is called vanishing intuitionistic fuzzy point(VIFP) in X.

Definition 5. [17]

(a) Let $c(\lambda, \delta)$ be an IFP in X such that $\lambda, \delta \in(0,1)$ and $A=\left\{\left\langle x, \mu_{A}(x), v_{A}(x)\right\rangle: x \in X\right\}$ be an IFS in X. $c(\lambda, \delta)$ is said to be properly contained in $\mathrm{A}\left(c(\lambda, \delta) \in A\right.$ for short) if $\lambda<\mu_{A}(c)$ and $\delta>\mu_{A}(c)$.

(b) Let $c(\delta)$ be a VIFS in X such that $\delta \in(0,1)$ and $A=\left\{\left\langle x, \mu_{A}(x), v_{A}(x)\right\rangle: x \in X\right\}$ be an IFS in X. $c(\delta)$ is said to be properly contained in $\mathrm{A}\left(c(\delta) \in A\right.$ for short) if $\mu_{A}(c)=0$ and $\delta>\mu_{A}(c)$.

Definition 6. [17]

(a) If $B=\left\{\left\langle y, \mu_{B}(y), v_{B}(y)\right\rangle: y \in Y\right\}$ is an IFS in $Y$ then the pre-image of $B$ under $f$ denoted by $f^{-1}(B)$, is an IFS in $X$ defined by

$$
f^{-1}(B)=\left\{\left\langle x, f^{-1}\left(\mu_{B}\right)(x), f^{-1}\left(v_{B}\right)(x)\right\rangle: x \in X\right\}
$$

(b) If $A=\left\{\left\langle x, \mu_{A}(x), v_{A}(x)\right\rangle: x \in X\right\}$ is an IFS in $\mathrm{X}$ then the image of $\mathrm{A}$ under $\mathrm{f}$ denoted by $\mathrm{f}(\mathrm{A})$, is an IFS in $\mathrm{Y}$ defined by

$$
f(A)=\left\{\left\langle y, f\left(\mu_{A}\right)(y), 1-f\left(1-v_{A}(y)\right)\right\rangle: y \in Y\right\}
$$

where

$$
\begin{gathered}
f\left(\mu_{A}\right)(y)= \begin{cases}\sup _{x \in f^{-1}(y)} \mu_{A}(x), & \text { if } f^{-1}(y) \neq \varnothing, \\
0, & \text { otherwise. }\end{cases} \\
1-f\left(1-v_{A}(y)\right)= \begin{cases}\inf _{x \in f^{-1}(y)} v_{A}(x), & \text { if } f^{-1}(y) \neq \varnothing, \\
1, & \text { otherwise. }\end{cases}
\end{gathered}
$$

Lemma 1. [17] Suppose $f: X \rightarrow Y$ is a function. If $c(\lambda, \delta)$ is an IFP in $X$ then $f(c(\lambda, \delta))$ is an IFP in $Y$ denoted by $c(f(\lambda, \delta))$. 
Definition 7. [17] Let $0_{\sim}=\{\langle x, 0,1\rangle: x \in X\}$ and $1_{\sim}=\{\langle x, 1,0\rangle: x \in X\}$. An intuitionistic fuzzy topology (IFT) on a nonempty set $\mathrm{X}$ is a family $\tau$ of IFSs in $\mathrm{X}$ satisfying the following axioms:

(T1) $0_{\sim}, 1_{\sim} \in \tau$,

(T2) $G_{1} \cap G_{2} \in \tau$ for any $G_{1}, G_{2} \in \tau$,

(T3) $\cup G_{i} \in \tau$ for any arbitrary family $\left\{G_{i}: i \in J\right\} \subseteq \tau$.

The pair $(X, \tau)$ is called an intuitionistic fuzzy topological space (IFTS) and IFS in $\tau$ is known as intuitionistic fuzzy open set (IFOS) in X. The complement $\bar{A}$ of an IFOS A in an IFTS $(\mathrm{X}, \tau)$ is called an intuitionistic fuzzy closed set (IFCS) in X.

Definition 8. [17] Let $(X, \tau)$ and $(Y, \sigma)$ be two IFTSs and let $f: X \rightarrow Y$ be a function. Then $f$ is said to be fuzzy continuous if and only if the pre-image of each IFS in $\sigma$ is IFS in $\tau$.

Definition 9. [17] Let $(X, \tau)$ and $(Y, \sigma)$ be two IFTSs and let $f: X \rightarrow Y$ be a function. Then $\mathrm{f}$ is said to be fuzzy open if and only if the image of each IFS in $\tau$ is IFS in $\sigma$.

Definition 10. [17] The $\mathrm{f}:(\mathrm{X}, \tau) \rightarrow(\mathrm{Y}, \sigma)$ is said to be fuzzy continuous if and only if the pre-image of each IFCS in $\sigma$ is IFCS in $\tau$.

Definition 11. [17] Let $(X, \tau)$ be an IFTS. Then

(a) $\mathrm{X}$ is said to be fuzzy $C_{5}$-disconnected if there exists an intuitionistic fuzzy open and fuzzy closed set $\mathrm{G}$ such that $\mathrm{G} \neq 1 \sim$ and $\mathrm{G} \neq 0 \sim$.

(b) $\mathrm{X}$ is said to be fuzzy $C_{5}$-connected if is not fuzzy $C_{5}$-disconnected.

Theorem 1. [17] Let $f:(X, \tau) \rightarrow(Y, \phi)$ be a fuzzy continuous surjection. If $(X, \tau)$ is fuzzy $C_{5}$-connected then so $(Y, \phi)$.

Definition 12. [17] Let $(X, T)$ be ordinary topological. The collection

$$
\tilde{\tau}=\left\{G: G \text { is an intuitionistic fuzzy set on } X \text { and } G_{\circ} \in T\right\}
$$

is an intuitionistic fuzzy topology on $X$ induced by $T$. The pair $(X, \tilde{\tau})$ is called the intuitionistic fuzzy topological space induced by $(X, T)$. Thus if $\varepsilon_{I}$ is the Euclidean subspace topology on $I$ then $\left(\mathrm{I}, \widetilde{\varepsilon}_{I}\right)$ denotes the IFTS induced by the (usual) topological space (I, $\left.\varepsilon_{I}\right)$.

Definition 13. [3] Let $(X, \tau)$ be an IFTS. If $\alpha:\left(I, \widetilde{\varepsilon}_{I}\right) \rightarrow(X, \tau)$ is a fuzzy continuous $E$ is a fuzzy $C_{5}$-connected in (I, $\left.\widetilde{\varepsilon_{I}}\right)$ with $\mu_{E}(0), \mu_{E}(1)>0$ and $v_{E}(0), v_{E}(1)<1$ then the IFS $\alpha(E)$ is called an intuitionistic path in $(\mathrm{X}, \tau)$. The intuitionistic fuzzy points $c_{1}(\lambda, \delta)$ and $c_{2}(\gamma, \theta)$ in $(\mathrm{X}, \tau)$ are called the initial point and the terminal point of the intuitionistic fuzzy path $\alpha(E)$ in respectively.

If the initial point equals to the terminal point, then we call it an intuitionistic path an intuitionistic fuzzy loop. The collection of all intuitionistic fuzzy loops in $(X, \tau)$ by $\Omega((X, \tau), c(\lambda, \delta))$ The IFP $c(\lambda, \delta)$ is called an intuitionistic fuzzy base point of $(X, \tau)$ and $((X, \tau), c(\lambda, \delta))$ is called pointed intuitionistic fuzzy space.

Lemma 2. [3] Let $(X, \tau)$ and $(Y, \sigma)$ be two IFTSs. Let the intuitionistic fuzzy sets $A$ and $B$ taking only values 0 and 1 on $X$, be closed IFS's in $(X, \tau)$ and $A \cup B=X$. Let $f:\left(A, \tau_{A}\right) \rightarrow(Y, \sigma)$ and $f:\left(B, \tau_{B}\right) \rightarrow(Y, \sigma)$ be two continuous functions. If $\left.f\right|_{A \cup B}=\left.g\right|_{A \cup B}$ then $h:(X, \tau) \rightarrow(Y, \sigma)$ defined by

$$
h(x)= \begin{cases}f(x), & \text { if } x \in A \\ g(x), & \text { if } x \in B\end{cases}
$$

is a fuzzy continuous function.

Lemma 3. [3] Let its $[a, b]=\{t: a \leq t \leq b\}$, topology taken as Euclidean subspace topology denoted by $\varepsilon_{[a, b]}$. If $\xi:\left([a, b], \varepsilon_{[a, b]}\right) \rightarrow\left(I, \varepsilon_{I}\right)$ is a (usual) homeomorphism, then

$$
\xi:\left([a, b], \widetilde{\varepsilon}_{[a, b]}\right) \rightarrow\left(I, \widetilde{\varepsilon}_{I}\right) .
$$


Lemma 4. [3] Let the intuitionistic fuzzy sets $E$ and $F$ be $C_{5}$-connected in the IFTS $\left(I, \widetilde{\varepsilon}_{I}\right)$ with $\mu_{E}(0), \mu_{E}(1)>0$, $v_{E}(0), v_{E}(1)<1$ and $\mu_{F}(0), \mu_{F}(1)>0, v_{F}(0), v_{F}(1)<1$. Then the intuitionistic fuzzy set $N$ defined by

$$
N(t)= \begin{cases}\mu_{E}(2 t), v_{E}(1-2 t), & \text { if } 0 \leq t \leq \frac{1}{2} \\ \mu_{F}(2 t-1), v_{F}(2-2 t), & \text { if } \frac{1}{2} \leq t \leq 1\end{cases}
$$

is $C_{5}$-connected with $\mu_{N}(0), \mu_{N}(1)>0, v_{N}(0), v_{N}(1)<1$.

Theorem 2. [3] Let $c_{1}(\lambda, \delta), c_{2}(\gamma, \theta)$ and $c_{3}(\omega, \eta)$ intuitionistic fuzzy points in an IFTS $(X, \tau)$. If there exist an intuitionistic fuzzy topological path in $(X, \tau)$ with initial point $c_{1}(\lambda, \delta)$ and terminal point $c_{2}(\gamma, \theta)$ and there is an intuitionistic fuzzy path in $(X, \tau)$ with initial point $c_{2}(\gamma, \theta)$ and terminal point $c_{3}(\omega, \eta)$ then there is an intuitionistic fuzzy path in $(X, \tau)$ with initial point $c_{1}(\lambda, \delta)$ and terminal point $c_{3}(\omega, \eta)$.

Definition 14. [3] Let $\alpha, \beta:\left(I, \widetilde{\varepsilon}_{I}\right) \rightarrow(X, \tau)$ be fuzzy continuous functions and let the IFS's A, B be connected in $\left(\mathrm{I}, \widetilde{\varepsilon_{I}}\right)$ with $\mathrm{A}(\mathrm{i}, \mathrm{j}), \mathrm{B}(\mathrm{i}, \mathrm{j})$ are all positive for all $\mathrm{i}, \mathrm{j}=0,1$. Let $\alpha(A), \beta(B) \in \Omega((X, \tau), c(\lambda, \delta))$. We say $\alpha(A)$ and $\beta(B)$ are intuitionistic fuzzy homotopic modulo $c(\lambda, \delta)$ ) if there exist a fuzzy continuous function $\mathrm{H}$ : $\left(I, \widetilde{\varepsilon_{I}}\right) \times\left(I, \widetilde{\varepsilon_{I}}\right) \rightarrow(X, \tau)$ such that $\mathrm{H}(\mathrm{t}, 0)=\alpha\left(t_{A(t)}\right), \mathrm{H}(\mathrm{t}, 1)=\beta\left(t_{B(t)}\right)$ for all $\mathrm{t} \in I$,

$$
\left.H(0, s)=\alpha\left(0_{A(0)}\right)=\beta\left(0_{B(0)}\right)=c(\lambda, \delta)\right),
$$

and

$$
\left.H(1, s)=\alpha\left(1_{A(1)}\right)=\beta\left(1_{B(1)}\right)=c(\lambda, \delta)\right)
$$

for all $\mathrm{s} \in I$.

The function $\mathrm{H}$ is called an intuitionistic fuzzy homotopy modulo $c(\lambda, \delta))$ between $\alpha(A)$ and $\beta(B)$, and we write $\mathrm{H}: \alpha(A) \simeq_{c(\lambda, \delta))} \beta(B)$ or simply $\alpha(A) \simeq_{c(\lambda, \delta))} \beta(B)$.

Theorem 3. [3] The relation $\simeq_{c(\lambda, \delta))}$ on the set $\Omega((X, \tau), c(\lambda, \delta))$ is an equivalent relation.

Proposition 1. [3] Let $\alpha(A), \beta(B), \sigma(U), \rho(V) \in \Omega((X, \tau), c(\lambda, \delta))$ such that $\alpha(A) \simeq_{c(\lambda, \delta))} \sigma(U)$ and $\beta(B) \simeq_{c(\lambda, \delta))}$ $\rho(V)$. Then

$$
\alpha(A) * \beta(B) \simeq_{c(\lambda, \delta))} \sigma(U) * \rho(V) .
$$

Proposition 2. [3] Let $\alpha(A) \in \Omega((X, \tau), c(\lambda, \delta))$. Then there exist a continuous function $\bar{\alpha}:\left(I, \widetilde{\varepsilon_{I}}\right) \rightarrow(X, \tau)$ and a connected intuitionistic fuzzy set $D$ in $\left(I, \widetilde{\varepsilon_{I}}\right)$ such that $\bar{\alpha}(D) \in \Omega((X, \tau), c(\lambda, \delta))$.

Proposition 3. [3] Let $\alpha(A), \beta(B) \in \Omega((X, \tau), c(\lambda, \delta))$. If $\alpha(A) \simeq_{c(\lambda, \delta))} \beta(B)$, then $\bar{\alpha}(A) \simeq_{c(\lambda, \delta))} \bar{\beta}(B)$.

Definition 15. [3] Let $\alpha(A), \beta(B) \in \Omega((X, \tau), c(\lambda, \delta))$. A loop $\psi(N)$ defined by

$$
\psi(N)=(\alpha * \beta)(N)= \begin{cases}\alpha(2 t), & \text { if } 0 \leq t \leq \frac{1}{2} \\ \beta(2 t-1), & \text { if } \frac{1}{2} \leq t \leq 1\end{cases}
$$

is called the product of intuitionistic fuzzy loops $\alpha(A)$ and $\beta(B)$. For homotopic classes $[\alpha(A)]$ and $[\beta(B)]$ in $\pi_{1}((X, \tau), c(\lambda, \delta))$, we defined the product $[\alpha(A)] \cdot[\beta(B)]=[\alpha(A) \cdot \beta(B)]=[(\alpha * \beta)(N)]$.

Theorem 4. [3] The set $\pi_{1}((X, \tau), c(\lambda, \delta))$ form a group under the product operation.

Proof. The proof follows directly from the following lemmas.

Lemma 5. [3] The product operation - is associative i. e., $\forall[\alpha(A)],[\beta(B)],[\sigma(U)] \in \pi_{1}((X, \tau), c(\lambda, \delta))$,

$$
\{[\alpha(A)] \cdot[\beta(B)]\} \cdot[\sigma(U)]=[\alpha(A)] \cdot\{[\beta(B)] \cdot[\sigma(U)]\} .
$$

Lemma 6. [3] The element $[\circ(E)]$ where $\circ(E)$ is a constant intuitionistic fuzzy loop defined by $\circ\left(t_{E(t)}\right)=c(\lambda, \delta)$, for all $t \in I$ is the identity of $\pi_{1}((X, \tau), c(\lambda, \delta))$. 
Lemma 7. [3] For each $[\alpha(A)] \in \pi_{1}((X, \tau), c(\lambda, \delta))$,

$$
[\alpha(A)] \cdot[\bar{\alpha}(D)]=[\circ(E)]=[\bar{\alpha}(D)] \cdot[\alpha(A)] .
$$

\section{Main results}

\subsection{Necessary and sufficient conditions for a fundamental group of IFTSs to be abelian}

In this section, we characterized the necessary and sufficient conditions for a fundamental group of IFTS to be abelian. As it is generally described in [18] that a group $G$ is said to be abelian if it is commutative. Further applications includes; the unitary character group of abelian unipotent groups [19] and a theorem on the action of abelian unitary groups in [20].

Now, follow from the above descriptions, it is discovered that the study of abelian fundamental group of IFTSs is very essential. Therefore we have the following definition and theorem:

Definition 16. Let $[\alpha(A)]$ and $[\beta(B)]$ be elements of $\pi_{1}((X, \tau), c(\lambda, \delta))$, then $\pi_{1}((X, \tau), c(\lambda, \delta))$ is called abelian fundamental group of intuitionistic fuzzy topological space if

$$
[(\alpha * \beta)(F)]=[(\beta * \alpha)(H)]
$$

for some connected IFSs $\mathrm{F}$ and $\mathrm{H}$ in $\left(I, \tilde{\varepsilon}_{I}\right)$.

Theorem 5. Let $[\alpha(A)],[\beta(B)] \in \pi_{1}((X, \tau), c(\lambda, \delta))$ and $[o(E)]$ be identity in $\pi_{1}((X, \tau), c(\lambda, \delta))$. Then $\pi_{1}((X, \tau), c(\lambda, \delta))$ is abelian iff $(\alpha * o)(L) *(o * \beta)(M) \simeq_{c(\lambda, \delta)}(o * \alpha)(N) *(\beta * o)(P)$ for some connected IFSs $L, M, N$ and $P$ in $\left(I, \tilde{\varepsilon}_{I}\right)$.

Proof. $(\Rightarrow)$ Let $\pi_{1}((X, \tau), c(\lambda, \delta))$ be abelian fundamental group of intuitionistic fuzzy topological space and $[\alpha(A)],[\beta(B)]$ and $[o(E)] \in \pi_{1}((X, \tau), c(\lambda, \delta))$.

Goal: We will show that $(\alpha * o)(\mathrm{L}) *(o * \beta)(\mathrm{M}) \simeq_{c(\lambda, \delta)}(o * \alpha)(\mathrm{N}) *(\beta * o)(\mathrm{P})$ for some connected IFSs $\mathrm{L}, \mathrm{M}, \mathrm{N}$ and $\mathrm{P}$ in $\left(I, \tilde{\varepsilon}_{I}\right)$.

To show this, it is suffices to show that there exists loop $\sigma(C)$ such that:

(i) $\sigma(C) \simeq_{c(\lambda, \delta)}(\alpha * o)(\mathrm{L}) *(o * \beta)(\mathrm{M})$

(ii) $\sigma(\mathrm{C}) \simeq_{c(\lambda, \delta)}(o * \alpha)(\mathrm{N}) *(\beta * o)(\mathrm{P})$

For (i): The function $H_{1}:\left(I, \widetilde{\varepsilon_{I}}\right) \times\left(I, \widetilde{\varepsilon_{I}}\right) \rightarrow(X, \tau)$ defined by

$$
H_{1}(t, s)= \begin{cases}\alpha\left(((t)(s)+(2 t)(1-s))_{A((t)(s)+(2 t)(1-s))),}\right. & \text { if } 0 \leq t \leq \frac{1+s}{2} \\ c(\lambda, \delta)=o\left(t_{E(t)}\right), & \text { if } \frac{1+s}{2} \leq t \leq 1\end{cases}
$$

is a homotopy between $\alpha(A)$ and $(\alpha * o)(\mathrm{L})$ i. e., $H_{1}$ is continuous by Lemma 2,

$$
\begin{aligned}
H_{1}(t, 0) & = \begin{cases}\alpha\left((2 t)_{A(2 t)}\right), & \text { if } 0 \leq t \leq \frac{1+s}{2} \\
c(\lambda, \delta)=o\left(t_{E(t)}\right), & \text { if } \frac{1+s}{2} \leq t \leq 1\end{cases} \\
& =(\alpha * o)\left(t_{L(t)}\right),
\end{aligned}
$$

$H_{1}(\mathrm{t}, 1)=\alpha\left(t_{A(t)}\right)$ and $H_{1}(0, \mathrm{~s})=\alpha\left(0_{A(0)}\right)=c(\lambda, \delta)=o\left(1_{E(1)}\right)=H_{1}(1, \mathrm{~s})$. Then $\alpha(A) \simeq_{c(\lambda, \delta)}(\alpha * o)(\mathrm{L})$.

Also, the function $H_{2}:\left(I, \widetilde{\varepsilon_{I}}\right) \times\left(I, \widetilde{\varepsilon_{I}}\right) \rightarrow(X, \tau)$ defined by

$$
H_{2}(t, s)= \begin{cases}c(\lambda, \delta)=o\left(t_{E(t)}\right), & \text { if } 0 \leq t \leq \frac{1-s}{2} \\ \beta\left(((t)(s)+(2 t-1)(1-s))_{B((t)(s)+(2 t-1)(1-s))}\right), & \text { if } \frac{1-s}{2} \leq t \leq 1\end{cases}
$$


is a homotopy between $\beta(B)$ and $(o * \beta)(\mathrm{M})$ i. e., $\mathrm{H}_{2}$ is continuous by Lemma 2,

$$
\begin{aligned}
H_{2}(t, 0) & = \begin{cases}c(\lambda, \delta)=o\left(t_{E(t)}\right), & \text { if } 0 \leq t \leq \frac{1-s}{2} \\
\beta\left((2 t-1)_{B(2 t-1)}\right), & \text { if } \frac{1-s}{2} \leq t \leq 1\end{cases} \\
& =(o * \beta)\left(\left(t_{M(t)}\right)\right),
\end{aligned}
$$

$H_{2}(\mathrm{t}, 1)=\beta\left(t_{B(t)}\right)$ and $H_{2}(0, \mathrm{~s})=c(\lambda, \delta)=o\left(0_{E(0)}\right)=\beta\left(1_{B(1)}\right)=H_{2}(1, \mathrm{~s})$, then $\beta(B) \simeq_{c(\lambda, \delta)}(o * \beta)(\mathrm{M})$. Thus, the function $\mathrm{H}:\left(I, \widetilde{\varepsilon_{I}}\right) \times\left(I, \widetilde{\varepsilon_{I}}\right) \rightarrow(X, \tau)$ defined by $\mathrm{H}(\mathrm{t}, \mathrm{s})=H_{1}(\mathrm{t}, \mathrm{s}) * H_{2}(\mathrm{t}, \mathrm{s})$ is a homotopy between $\sigma$ and $(\alpha * o)(\mathrm{L}) *(o * \beta)(\mathrm{M})$ i. e., $\mathrm{H}$ is the composition of two continuous functions, $\mathrm{H}(\mathrm{t}, 0)=(\alpha * o)(\mathrm{L}) *(o * \beta)(\mathrm{M}), \mathrm{H}(\mathrm{t}$, $1)=(\alpha * \beta)(\mathrm{F})=\sigma(\mathrm{C})$ and $\mathrm{H}(0, \mathrm{~s})=H_{1}(0, \mathrm{~s}) * H_{2}(0, \mathrm{~s})=H_{1}(1, \mathrm{~s}) * H_{2}(1, \mathrm{~s})=\mathrm{H}(1, \mathrm{~s})$. Therefore $\sigma(\mathrm{C}) \simeq_{c(\lambda, \delta)}(\alpha * o)(\mathrm{L})$ $*(o * \beta)(\mathrm{M})$.

For (ii): $K_{1}:\left(I, \widetilde{\varepsilon_{I}}\right) \times\left(I, \widetilde{\varepsilon_{I}}\right) \rightarrow(X, \tau)$ defined by

$$
K_{1}(t, s)= \begin{cases}c(\lambda, \delta)=o\left(t_{E(t)}\right), & \text { if } 0 \leq t \leq \frac{1-s}{2} \\ \alpha\left(((t)(s)+(2 t-1)(1-s))_{A((t)(s)+(2 t-1)(1-s))}\right), & \text { if } \frac{1-s}{2} \leq t \leq 1\end{cases}
$$

is a homotopy between $\alpha(A)$ and $(o * \alpha)(\mathrm{N})$ i. e., $K_{1}$ is continuous by Lemma 2 ,

$$
\begin{aligned}
K_{1}(t, 0) & = \begin{cases}c(\lambda, \delta)=o\left(t_{E(t)}\right), & \text { if } 0 \leq t \leq \frac{1-s}{2} \\
\alpha\left((2 t-1)_{A(2 t-1)}\right), & \text { if } \frac{1-s}{2} \leq t \leq 1\end{cases} \\
& =(o * \beta)\left(\left(t_{M(t)}\right)\right),
\end{aligned}
$$

$K_{1}(\mathrm{t}, 1)=\alpha\left(t_{A(t)}\right)$ and $K_{1}(0, \mathrm{~s})=c(\lambda, \delta)=o\left(0_{E(0)}\right)=\alpha\left(1_{A(1)}\right)=K_{1}(1, \mathrm{~s})$, then $\alpha(A) \simeq_{c(\lambda, \delta)}(o * \alpha)(\mathrm{N})$.

Also the function $K_{2}:\left(I, \widetilde{\varepsilon_{I}}\right) \times\left(I, \widetilde{\varepsilon_{I}}\right) \rightarrow(X, \tau)$ defined by

$$
K_{2}(t, s)= \begin{cases}\beta\left(((t)(s)+(2 t)(1-s))_{B((t)(s)+(2 t)(1-s))),}\right. & \text { if } 0 \leq t \leq \frac{1+s}{2} \\ c(\lambda, \delta)=o\left(t_{E(t)}\right), & \text { if } \frac{1+s}{2} \leq t \leq 1\end{cases}
$$

is a homotopy between $\beta(B)$ and $(\beta * o)(P)$ i. e., $K_{2}$ is continuous by Lemma 2,

$$
\begin{aligned}
K_{2}(t, 0) & = \begin{cases}\beta\left((2 t)_{B(2 t)}\right), & \text { if } 0 \leq t \leq \frac{1+s}{2} \\
c(\lambda, \delta)=o\left(t_{E(t)}\right), & \text { if } \frac{1+s}{2} \leq t \leq 1\end{cases} \\
& =(\beta * o)\left(t_{P(t)}\right),
\end{aligned}
$$

$K_{2}(\mathrm{t}, 1)=\beta\left(t_{B(t)}\right)$ and $K_{2}(0, \mathrm{~s})=\beta\left(0_{B(0)}\right)=c(\lambda, \delta)=o\left(1_{E(1)}\right)=K_{2}(1, \mathrm{~s})$. Then $\alpha(A) \simeq_{c(\lambda, \delta)}(\beta * o)(\mathrm{P})$. Hence the function $\mathrm{K}::\left(I, \widetilde{\varepsilon_{I}}\right) \times\left(I, \widetilde{\varepsilon_{I}}\right) \rightarrow(X, \tau)$ defined by $\mathrm{K}(\mathrm{t}, \mathrm{s})=K_{1}(\mathrm{t}, \mathrm{s}) * K_{2}(\mathrm{t}, \mathrm{s})$ is a homotopy between $\sigma(C)$ and $(o * \alpha)(\mathrm{N}) *(\beta * o)(\mathrm{P}) i$. $e ., \mathrm{K}$ is the composition of two continuous functions, $\mathrm{K}(\mathrm{t}, 0)=\left(\varepsilon_{p} * \mathrm{f}\right) *\left(\mathrm{f} * \varepsilon_{p}\right)(\mathrm{t}), \mathrm{K}(\mathrm{t}$, $1)=(\mathrm{f} * \mathrm{~g})(\mathrm{t})=\sigma(\mathrm{t})$ and $\mathrm{K}(0, \mathrm{t})=K_{1}(0, \mathrm{~s}) * K_{2}(0, \mathrm{~s})=K_{1}(1, \mathrm{~s}) * K_{2}(1, \mathrm{~s})=\mathrm{K}(1, \mathrm{x})$. Then $\sigma(C) \simeq_{c(\lambda, \delta)}(o * \alpha)(\mathrm{N}) *$ $(\beta * o)(\mathrm{P})$. Thus, the result follows.

$(\Leftarrow)$ Suppose $(\alpha * o)(\mathrm{L}) *(o * \beta)(\mathrm{M}) \simeq_{c(\lambda, \delta)}(o * \alpha)(\mathrm{N}) *(\beta * o)(\mathrm{P})$ for some connected IFSs L, M, L and P in $\left(I, \tilde{\varepsilon}_{I}\right)$,

since $(\alpha * \beta)(\mathrm{F})=(\alpha * o)(\mathrm{L}) *(o * \beta)(\mathrm{M}) \simeq_{c(\lambda, \delta)}(o * \alpha)(\mathrm{N}) *(\beta * o)(\mathrm{P})=(\beta * \alpha)(\mathrm{H})$ for some connected IFSs L, M, $\mathrm{L}$ and $\mathrm{P}$ in $\left(I, \tilde{\varepsilon}_{I}\right)$. Then $(\alpha * \beta)(\mathrm{F}) \simeq_{c(\lambda, \delta)}(\beta * \alpha)(\mathrm{H})$ which implies $[(\alpha * \beta)(\mathrm{F})]=[(\beta * \alpha)(\mathrm{H})]$ and by the concatenation of loop defined on $\pi_{1}(X, p)$, we have $[\alpha(A)] \cdot[\beta(B)]=[\beta(B)] \cdot[\alpha(A)]$. Thus, the result follows.

\subsection{Necessary and sufficient conditions for a subset of fundamental group of IFTSs to be a subgroup}

In this section, we characterized the necessary and sufficient conditions for a subset of fundamental group to be its subgroups. A necessary and sufficient conditions for a subset of a group to be a subgroup have been explained in [21], that is given a group $G$ with a particular binary operation, if a non-empty subset $H$ of $G$ is closed and every elements in it has inverse under that particular operation, then we say $H$ is a subgroup of $G$. Further applications includes; the maximal subgroups of symplectic groups stabilizing spreads [22]; 3-permutable subgroups and p-nilpotency of finite group II [23]; fuzzy subgroups of nilpotent groups [24]; subgroup of nilpotent group [25]; two theorems about nilpotent subgroup [26]. 
Now, follow from the above descriptions, it is discovered that the study of subgroups of fundamental group is very essential. Therefore we have the following definition:

Definition 17. Let $\pi_{1}((X, \tau), c(\lambda, \delta))$ be fundamental group of IFTS $X$ and $U$ be nonempty subspace of $X$. If $\pi_{1}((U, \tau), c(\lambda, \delta))$ is a group under the operation $\cdot$ of $\pi_{1}((X, \tau), c(\lambda, \delta))$, then $\pi_{1}((U, \tau), c(\lambda, \delta))$ is a subgroup of $\pi_{1}((X, \tau), c(\lambda, \delta))$.

Theorem 6. Let $\pi_{1}((X, \tau), c(\lambda, \delta))$ be fundamental group of IFTS $X$ and $U$ be nonempty subspace of $X$. Then $\pi_{1}((U, \tau), c(\lambda, \delta))$ is a subgroup of $\pi_{1}((X, \tau), c(\lambda, \delta))$ if and only if the following conditions hold

(i) $(\alpha * o)(L) \simeq_{c(\lambda, \delta)} \alpha(A) \simeq_{c(\lambda, \delta)}(o * \alpha)(N) \forall[\alpha(A)] \in \pi_{1}(U, p)$, where $[o(E)]$ is the identity in $\pi_{1}((U, \tau), c(\lambda, \delta))$ and for some connected IFSs $A, E, L$ and $N \in\left(I, \tilde{\varepsilon}_{I}\right)$.

(ii) there exists $[\alpha(A)] \in \pi_{1}((U, \tau), c(\lambda, \delta))$ such that $\left.[\beta(B)] \cdot[\sigma(C)]=[\alpha(A)] \forall[\beta(B)],[\sigma(C))\right] \in$ $\pi_{1}((U, \tau), c(\lambda, \delta))$ and for some connected IFSs $A, B$ and $C \in\left(I, \tilde{\varepsilon}_{I}\right)$.

(iii) $\alpha(A) * \bar{\alpha}(J) \simeq_{c(\lambda, \delta)} o(E) \simeq_{c(\lambda, \delta)} \bar{\alpha}(J) * \alpha(A) \forall[\alpha(A)] \in \pi_{1}((U, \tau), c(\lambda, \delta))$, where $[o(E)],[\bar{\alpha}(J)]$ are the identity and inverse in $\pi_{1}((U, \tau), c(\lambda, \delta))$ respectively and for some connected IFSs $A, E$ and $J \in\left(I, \tilde{\varepsilon}_{I}\right)$.

Proof. Suppose $\pi_{1}((U, \tau), c(\lambda, \delta))$ is a subgroup of $\pi_{1}((X, \tau), c(\lambda, \delta))$

Goal: We will show that the three conditions above satisfied.

For (i): It is suffices to show that the following conditions are satisfied

(a) $\alpha(\mathrm{A}) \simeq_{c(\lambda, \delta)}(\alpha * o)(\mathrm{L})$

(b) $\alpha(\mathrm{A}) \simeq_{c(\lambda, \delta)}(o * \alpha)(\mathrm{N})$.

Consider the function $\alpha$ depend on (I, $\left.\tilde{\varepsilon}_{I}\right)$ to $(X, \tau)$ such that $\alpha\left(\left(I, \tilde{\varepsilon}_{I}\right)\right) \subseteq(U, \tau) \subseteq(X, \tau)$ where $\mathrm{U}$ is a subspace of $X$. Thus $\alpha(A)$ depend on $\left(\mathrm{I}, \tilde{\varepsilon}_{I}\right)$ to $(U, \tau)$.

For (a): The function $\mathrm{H}:\left(I, \widetilde{\varepsilon_{I}}\right) \times\left(I, \widetilde{\varepsilon_{I}}\right) \rightarrow(X, \tau)$ defined by

$$
H(t, s)= \begin{cases}\alpha\left(((t)(s)+(2 t)(1-s))_{A((t)(s)+(2 t)(1-s))}\right), & \text { if } 0 \leq t \leq \frac{1+s}{2} \\ c(\lambda, \delta)=o\left(t_{E(t)}\right), & \text { if } \frac{1+s}{2} \leq t \leq 1\end{cases}
$$

is a homotopy between $\alpha(A)$ and $(\alpha * o)(\mathrm{L})$ i. e., $\mathrm{H}$ is continuous by Lemma 2,

$$
\begin{aligned}
H(t, 0) & = \begin{cases}\alpha\left((2 t)_{A(2 t)}\right), & \text { if } 0 \leq t \leq \frac{1+s}{2} \\
c(\lambda, \delta)=o\left(t_{E(t)}\right), & \text { if } \frac{1+s}{2} \leq t \leq 1\end{cases} \\
& =(\alpha * o)\left(t_{L(t)}\right),
\end{aligned}
$$

$H_{1}(\mathrm{t}, 1)=\alpha\left(t_{A(t)}\right)$ and $H_{1}(0, \mathrm{~s})=\alpha\left(0_{A(0)}\right)=c(\lambda, \delta)=\alpha\left(1_{A(1)}\right)=H_{1}(1, \mathrm{~s})$. Then $\alpha(A) \simeq_{c(\lambda, \delta)}(\alpha * o)(\mathrm{L})$.

For $(\mathrm{b})$ : The function $\mathrm{K}:\left(I, \widetilde{\varepsilon_{I}}\right) \times\left(I, \widetilde{\varepsilon_{I}}\right) \rightarrow(X, \tau)$ defined by

$$
K(t, s)= \begin{cases}c(\lambda, \delta)=o\left(t_{E(t)}\right), & \text { if } 0 \leq t \leq \frac{1-s}{2} \\ \alpha\left(((t)(s)+(2 t-1)(1-s))_{A((t)(s)+(2 t-1)(1-s))),}\right. & \text { if } \frac{1-s}{2} \leq t \leq 1\end{cases}
$$

is a homotopy between $\alpha(A)$ and $(o * \alpha)(\mathrm{N})$ i. e., $K_{1}$ is continuous by Lemma 2 ,

$$
\begin{aligned}
K(t, 0) & = \begin{cases}c(\lambda, \delta)=o\left(t_{E(t)}\right), & \text { if } 0 \leq t \leq \frac{1-s}{2} \\
\alpha\left((2 t-1)_{A(2 t-1)}\right), & \text { if } \frac{1-s}{2} \leq t \leq 1\end{cases} \\
& =(o * \beta)\left(\left(t_{M(t)}\right)\right),
\end{aligned}
$$

$\mathrm{K}(\mathrm{t}, 1)=\alpha\left(t_{A(t)}\right)$ and $\mathrm{K}(0, \mathrm{~s})=c(\lambda, \delta)=o\left(0_{E(0)}\right)=\alpha\left(1_{A(1)}\right)=\mathrm{K}(1, \mathrm{~s})$, then $\alpha(A) \simeq_{c(\lambda, \delta)}(o * \alpha)(\mathrm{N})$.

For (ii): Let $[\beta(B)],[\sigma(C))] \in \pi_{1}((U, \tau), c(\lambda, \delta))$

Goal: To show that there is $[\alpha(A)] \in \pi_{1}((U, \tau), c(\lambda, \delta))$ such that $\left.[\beta(B)] \cdot[\sigma(C)]=[\alpha(A)] \forall[\beta(B)],[\sigma(C))\right] \in$ $\pi_{1}((U, \tau), c(\lambda, \delta))$ and for some connected IFSs A, B and $C \in\left(I, \tilde{\varepsilon}_{I}\right)$. 
To show this it is suffices to show that $\alpha(A) \simeq_{c(\lambda, \delta)} \beta(B) \cdot \sigma(C)$. Define $\alpha(A)$ by

$$
\alpha\left(t_{A(t)}\right)= \begin{cases}\beta\left(0_{A(0)}\right), & \text { if } t=0 \\ \sigma\left(0_{C(0)}\right), & \text { if } 0<t \leq 1\end{cases}
$$

and the composition of $\beta(B)$ and $\sigma(C)$ as

$$
(\beta * \sigma)\left(t_{G(t)}\right)= \begin{cases}\beta\left((2 t)_{B(2 t)}\right), & \text { if } 0 \leq t \leq \frac{1}{2} \\ \sigma\left((2 t-1)_{C(2 t-1)}\right), & \text { if } \frac{1}{2} \leq t \leq 1\end{cases}
$$

where $\beta\left((0)_{B(0)}\right)=\sigma\left((0)_{C(0)}\right)=\beta\left((1)_{B(1)}\right)=\sigma\left((1)_{C(1)}\right)$. Then he function $\mathrm{H}:\left(I, \widetilde{\varepsilon_{I}}\right) \times\left(I, \widetilde{\varepsilon_{I}}\right) \rightarrow(X, \tau)$ defined by

$$
H(t, s)= \begin{cases}\left.\beta((2 t)(1-s))_{B((2 t)(1-s))}\right), & \text { if } 0 \leq t \leq \frac{1-s}{2} \\ \sigma\left(((2 t-1)(1-s))_{C((2 t-1)(1-s))}\right), & \text { if } \frac{1-s}{2} \leq t \leq 1\end{cases}
$$

is a homotopy between $\alpha(A)$ and $(\beta * \sigma)(\mathrm{G})$ i. e., $\mathrm{H}$ is continuous by Lemma 2,

$$
\begin{aligned}
H(t, 0) & = \begin{cases}\beta\left((2 t)_{B(2 t)}\right), & \text { if } 0 \leq t \leq \frac{1}{2} \\
\sigma\left((2 t-1)_{C(2 t-1)}\right), & \text { if } \frac{1}{2} \leq t \leq 1\end{cases} \\
& =(\beta * \sigma)\left(\left(t_{G(t)}\right)\right),
\end{aligned}
$$

$\mathrm{H}(\mathrm{t}, 1)=\alpha\left(t_{A(t)}\right)$ and $\mathrm{H}(0, \mathrm{~s})=\beta\left(0_{B(0)}\right)=\sigma\left(1_{C(1)}\right)=\mathrm{H}(1, \mathrm{~s})$. Therefore $\alpha(A) \simeq_{c(\lambda, \delta)} \beta(B) \cdot \sigma(C)$ $\forall[\alpha(A)],[\beta(B)],[\sigma(C)] \in \pi_{1}((U, \tau), c(\lambda, \delta))$.

For (iii): To prove this it is suffices to show the following:

(a): $o(E) \simeq_{c(\lambda, \delta)}(\alpha)(\mathrm{A}) *(\bar{\alpha})(\mathrm{J})$

(b): $o(E) \simeq_{c(\lambda, \delta)}(\bar{\alpha})(\mathrm{J}) *(\alpha)(\mathrm{A})$

$\forall[\alpha(A)] \in \pi_{1}((U, \tau), c(\lambda, \delta))$, where $[o(E)],[\bar{\alpha}(\mathrm{J})]$ are the identity and inverse in $\pi_{1}((U, \tau), c(\lambda, \delta))$ respectively and for some connected IFSs A, E and $\mathrm{J} \in\left(I, \tilde{\varepsilon}_{I}\right)$.

For (a): We define the composition of $\alpha(A)$ and $\bar{\alpha}(J)$ as

$$
(\alpha * \bar{\alpha})(G)= \begin{cases}\alpha\left((2 t)_{A(2 t)}\right), & \text { if } 0 \leq t \leq \frac{1}{2} \\ \bar{\alpha}\left((2 t-1)_{J(2 t-1)}\right), & \text { if } \frac{1}{2} \leq t \leq 1\end{cases}
$$

where $\alpha\left(0_{A(0)}\right)=\bar{\alpha}\left(1_{J(1)}\right)=\alpha\left(1_{A(1)}\right)=\bar{\alpha}\left(0_{J(0)}\right)$.

Then the function $\mathrm{H}:\left(I, \widetilde{\varepsilon_{I}}\right) \times\left(I, \widetilde{\varepsilon_{I}}\right) \rightarrow(X, \tau)$ defined by

$$
H(t, s)= \begin{cases}\left.\alpha((2 t)(1-s))_{B((2 t)(1-s))}\right), & \text { if } 0 \leq t \leq \frac{1-s}{2} \\ \bar{\alpha}\left(((2 t-1)(1-s))_{C((2 t-1)(1-s))),}\right. & \text { if } \frac{1-s}{2} \leq t \leq 1\end{cases}
$$

is a homotopy between $o(E)$ and $(\alpha * \bar{\alpha})(\mathrm{G})$ i. e., $\mathrm{H}$ is continuous by Lemma 2,

$$
\begin{aligned}
H(t, 0) & = \begin{cases}\alpha\left((2 t)_{A(2 t)}\right), & \text { if } 0 \leq t \leq \frac{1}{2} \\
\bar{\alpha}\left((2 t-1)_{J(2 t-1)}\right), & \text { if } \frac{1}{2} \leq t \leq 1\end{cases} \\
& =(\alpha * \bar{\alpha})\left(\left(t_{G(t)}\right)\right),
\end{aligned}
$$

$\mathrm{H}(\mathrm{t}, 1)=o\left(t_{E(t)}\right)$ and $\mathrm{H}(0, \mathrm{~s})=\alpha\left(0_{A(0)}\right)=\bar{\alpha}\left(s_{A(s)}\right)=\mathrm{H}(1, \mathrm{~s})$. Therefore $o(E) \simeq_{c(\lambda, \delta)}(\alpha * \bar{\alpha})(\mathrm{G})$ $\forall[\alpha(A)],[\bar{\alpha}(J)],[o(E)] \in \pi_{1}((U, \tau), c(\lambda, \delta))$.

For (b): We define the composition of $\bar{\alpha}(J)$ and $\alpha(A)$ as

$$
(\bar{\alpha} * \alpha)(G)= \begin{cases}\bar{\alpha}\left((2 t)_{J(2 t)}\right), & \text { if } 0 \leq t \leq \frac{1}{2} \\ \alpha\left((2 t-1)_{A(2 t-1)}\right), & \text { if } \frac{1}{2} \leq t \leq 1\end{cases}
$$

where $\alpha\left(0_{A(0)}\right)=\bar{\alpha}\left(1_{J(1)}\right)=\alpha\left(1_{A(1)}\right)=\bar{\alpha}\left(0_{J(0)}\right)$. 
Then the function $\mathrm{H}:\left(I, \widetilde{\varepsilon_{I}}\right) \times\left(I, \widetilde{\varepsilon_{I}}\right) \rightarrow(X, \tau)$ defined by

$$
H(t, s)= \begin{cases}\left.\bar{\alpha}((2 t)(1-s))_{J((2 t)(1-s))}\right), & \text { if } 0 \leq t \leq \frac{1-s}{2} \\ \alpha\left(((2 t-1)(1-s))_{A((2 t-1)(1-s))}\right), & \text { if } \frac{1-s}{2} \leq t \leq 1\end{cases}
$$

is a homotopy between $o(E)$ and $(\bar{\alpha} * \alpha)(\mathrm{G})$ i. e., $\mathrm{H}$ is continuous by Lemma 2 ,

$$
\begin{aligned}
H(t, 0) & = \begin{cases}\bar{\alpha}\left((2 t)_{J(2 t)}\right), & \text { if } 0 \leq t \leq \frac{1}{2} \\
\alpha\left((2 t-1)_{A(2 t-1)}\right), & \text { if } \frac{1}{2} \leq t \leq 1\end{cases} \\
& =(\bar{\alpha} * \alpha)\left(t_{G(t)}\right),
\end{aligned}
$$

$\mathrm{H}(\mathrm{t}, 1)=o\left(t_{E(t)}\right)$ and $\mathrm{H}(0, \mathrm{~s})=\bar{\alpha}\left(0_{J(0)}\right)=\alpha\left(1_{A(1)}\right)=\mathrm{H}(1, \mathrm{~s})$. Then $o(E) \simeq_{c(\lambda, \delta)}(\bar{\alpha} * \alpha)(\mathrm{G})$. Therefore $(\alpha)(\mathrm{A}) *$ $(\bar{\alpha})(\mathrm{J}) \simeq_{c(\lambda, \delta)} o(E) \simeq_{c(\lambda, \delta)}(\bar{\alpha})(\mathrm{J}) *(\alpha)(\mathrm{A}) \forall[\alpha(A)],[\bar{\alpha}(J)],[o(E)] \in \pi_{1}((U, \tau), c(\lambda, \delta))$.

$(\Leftarrow)$ Suppose the three conditions above satisfied.

Goal: We will show that $\pi_{1}((U, \tau), c(\lambda, \delta))$ is an IF subgroup of the fundamental group of IFTS $\pi_{1}((X, \tau), c(\lambda, \delta))$.

From (i): Since $(\alpha * o)(\mathrm{L}) \simeq_{c(\lambda, \delta)}(\alpha)(\mathrm{A}) \simeq_{c(\lambda, \delta)}(o * \alpha)(\mathrm{N})$ then $[(\alpha * o)(\mathrm{L})]=[\alpha(\mathrm{A})]=[(o * \alpha)(\mathrm{N})]$, which implies $[\alpha(\mathrm{A})] \cdot[o(E)]=[\alpha(\mathrm{A})]=[o(E)] \cdot[\alpha(\mathrm{A})]$. Therefore the identity $[o(E)]$ exist in $\pi_{1}((U, \tau), c(\lambda, \delta)) \forall$ $[\alpha(A)] \in \pi_{1}(\mathrm{U}, \mathrm{p})$, and for some connected IFSs $\mathrm{A}, \mathrm{E}, \mathrm{L}$ and $\mathrm{N} \in\left(I, \tilde{\varepsilon}_{I}\right)$.

From (ii): Since $(\beta * \sigma)(\mathrm{L}) \simeq_{c(\lambda, \delta)} \alpha(\mathrm{A})$ then we have $[(\beta * \sigma)(\mathrm{L})]=[\alpha(\mathrm{A})]$, which implies $[\beta(\mathrm{B})] \cdot[\sigma(\mathrm{C})]$ $=[\alpha(\mathrm{A})] \in \pi_{1}((U, \tau), c(\lambda, \delta)) \forall[\beta(B)],[\sigma(C)] \in \pi_{1}((U, \tau), c(\lambda, \delta))$ and for some connected IFSs A, B and C $\in\left(I, \tilde{\varepsilon}_{I}\right)$. Therefore $\pi_{1}((U, \tau), c(\lambda, \delta))$ is closed.

From (iii): Since $(\alpha)(\mathrm{A}) *(\bar{\alpha})(\mathrm{J}) \simeq_{c(\lambda, \delta)} o(E) \simeq_{c(\lambda, \delta)}(\bar{\alpha})(\mathrm{J}) *(\alpha)(\mathrm{A})$ then we have $[\alpha(\mathrm{A}) * \bar{\alpha}(\mathrm{J})]=[o(E)]$ $=[\bar{\alpha}(\mathrm{J}) * \alpha(\mathrm{A})]$, which implies $[\alpha(\mathrm{A})] \cdot[\bar{\alpha}(\mathrm{J})]=[o(E)]=[\bar{\alpha}(\mathrm{J})] \cdot[\alpha(\mathrm{A})]$. Therefore the inverse $[\bar{\alpha}(\mathrm{J})]$ exists in $\pi_{1}((U, \tau), c(\lambda, \delta)) \forall[\alpha(A)],[o(E)] \in \pi_{1}((U, \tau), c(\lambda, \delta))$. Now, clearly from the above $\pi_{1}((U, \tau), c(\lambda, \delta))$ is a Intuitionistic fuzzy subgroup of a fundamental group $\pi_{1}((X, \tau), c(\lambda, \delta))$.

\subsection{Necessary and sufficient condition for a subgroup of fundamental group of IFTSs to be normal}

Definition 18. Let $\pi_{1}((U, \tau), c(\lambda, \delta))$ be a subgroup of a fundamental group of IFTS $\pi_{1}((X, \tau), c(\lambda, \delta))$, then

(i) for $[\alpha(A)] \in \pi_{1}((X, \tau), c(\lambda, \delta))$, the subset $[\alpha(A)]\left(\pi_{1}((U, \tau), c(\lambda, \delta))\right)$ of $\pi_{1}((X, \tau), c(\lambda, \delta))$ is called the left coset of $\pi_{1}((U, \tau), c(\lambda, \delta))$ determined by $[\alpha(A)]$ i.e.

$$
[\alpha(A)]\left(\pi_{1}((U, \tau), c(\lambda, \delta))\right)=\left\{[\alpha(A)] \cdot[\beta(B)]:[\beta(B)] \in \pi_{1}((U, \tau), c(\lambda, \delta))\right\} .
$$

(ii) for $[\alpha(A)] \in \pi_{1}((X, \tau), c(\lambda, \delta))$ the subset $\left(\pi_{1}((U, \tau), c(\lambda, \delta))\right)[\alpha(A)]$ of $\pi_{1}((X, \tau), c(\lambda, \delta))$ is called the right coset of $\pi_{1}((U, \tau), c(\lambda, \delta))$ determined by $[\alpha(A)]$ i. e.

$$
\left(\pi_{1}((U, \tau), c(\lambda, \delta))\right)[\alpha(A)]=\left\{[\beta(B)] \cdot[\alpha(A)]:[\beta(B)] \in \pi_{1}((X, \tau), c(\lambda, \delta))\right\} .
$$

Definition 19. A subgroup $\pi_{1}((U, \tau), c(\lambda, \delta))$ of a fundamental group of IFTS $\pi_{1}((X, \tau), c(\lambda, \delta))$ is called normal if its left and right coset coincide i. e. $[\alpha(A)]\left(\pi_{1}((U, \tau), c(\lambda, \delta))\right)=\left(\pi_{1}((U, \tau), c(\lambda, \delta))\right)[\alpha(A)]$ for $[\alpha(A)]$ $\in \pi_{1}((U, \tau), c(\lambda, \delta))$ written as

$$
\pi_{1}((U, \tau), c(\lambda, \delta)) \triangle \pi_{1}((X, \tau), c(\lambda, \delta))
$$

Theorem 7. Let $\pi_{1}((U, \tau), c(\lambda, \delta))$ be an intuitionistic fuzzy subgroup of $\pi_{1}((X, \tau), c(\lambda, \delta))$. Then $\pi_{1}((U, \tau), c(\lambda, \delta))$ $\triangle \pi_{1}((X, \tau), c(\lambda, \delta))$ if and only if $[(\alpha * \beta)(D) * \bar{\alpha}(J)] \in \pi_{1}((U, \tau), c(\lambda, \delta))$ i. e. iff $\exists\left[\beta^{\prime}(B)\right] \in \pi_{1}((U, \tau), c(\lambda, \delta))$ such that $[(\alpha * \beta)(D) * \bar{\alpha}(J)]=\left[\beta^{\prime}(B)\right] \forall[\alpha(A)],[\bar{\alpha}(J)] \in \pi_{1}((X, \tau), c(\lambda, \delta))$ and $[\beta(B)] \in \pi_{1}((U, \tau), c(\lambda, \delta))$.

Proof. $(\Rightarrow)$ : Let $\pi_{1}((U, \tau), c(\lambda, \delta))$ be an intuitionistic fuzzy subgroup of $\pi_{1}((X, \tau), c(\lambda, \delta))$ and $\pi_{1}((U, \tau), c(\lambda, \delta)) \triangle \pi_{1}((X, \tau), c(\lambda, \delta))$ for all $[\beta(B)] \in \pi_{1}((U, \tau), c(\lambda, \delta))$ and $[\alpha(A)],[\bar{\alpha}(J)] \in \pi_{1}((X, \tau), c(\lambda, \delta))$ (where $[\bar{\alpha}(J)]$ is the inverse of $[\alpha(A)])$.

Goal: We are to show that $\exists[(\alpha * \beta)(D) * \bar{\alpha}(J)] \in \pi_{1}((U, \tau), c(\lambda, \delta))$. 
To show this, it is suffices to show that $\exists\left[\beta^{\prime}(B)\right] \in \pi_{1}((U, \tau), c(\lambda, \delta))$ such that $[(\alpha * \beta)(D) * \bar{\alpha}(J)]=\left[\beta^{\prime}(B)\right]$ i. e., $(\alpha * \beta) * \bar{\alpha}(J) \simeq_{c(\lambda, \delta)} \beta^{\prime}(B)$. Consider the composition of functions $\alpha(A), \beta(B)$ and $\bar{\alpha}(J)$ defined below

$$
(\alpha * \beta * \bar{\alpha})(t)= \begin{cases}\alpha\left((3 t)_{A(3 t)}\right), & \text { if } 0 \leq t \leq \frac{1}{3} \\ \beta\left((3 t-1)_{B(3 t-1)}\right), & \text { if } \frac{1}{3} \leq t \leq \frac{2}{3} \\ \bar{\alpha}\left((3 t-2)_{J(3 t-2)}\right), & \text { if } \frac{2}{3} \leq t \leq 1\end{cases}
$$

where $\alpha\left(0_{A(0)}\right)=\beta\left(0_{B(0)}\right)=\bar{\alpha}\left(0_{J(0)}\right)=\alpha\left(1_{A(1)}\right)=\beta\left(1_{B(1)}\right)=\bar{\alpha}\left(1_{J(1)}\right)$. Then we can define the homotopy between $(\alpha * \beta) * \bar{\alpha}(J)$ and $\beta^{\prime}(B)$ as a function $\mathrm{H}:\left(I, \widetilde{\varepsilon_{I}}\right) \times\left(I, \widetilde{\varepsilon_{I}}\right) \rightarrow(X, \tau)$ by

$$
H(t, s)= \begin{cases}\alpha\left(((3 t)(s))_{A((3 t)(s))}\right), & \text { if } 0 \leq t \leq \frac{s}{3} \\ \beta\left(((3 t-1)(s)+(t)(1-s))_{B((3 t-1)(s)+(t)(1-s))),}\right. & \text { if } \frac{s}{3} \leq t \leq \frac{3-s}{3} \\ \bar{\alpha}\left(((3 t-2)(s))_{J((3 t-2)(s))}\right), & \text { if } \frac{3-s}{3} \leq t \leq 1\end{cases}
$$

i. e., $\mathrm{H}$ is continuous by Lemma 2 ,

$$
\begin{aligned}
H(t, 0) & = \begin{cases}\alpha\left((0)_{A(0)}\right), & \text { if } 0 \leq t \leq 0 \\
\beta\left((t)_{B(t)}\right), & \text { if } 0 \leq t \leq 1 \\
\bar{\alpha}\left((0)_{J(0)}\right), & \text { if } 1 \leq t \leq 1\end{cases} \\
& =\beta^{\prime}\left((t)_{B(t)}\right),
\end{aligned}
$$

$\mathrm{H}(\mathrm{t}, 1)=(\alpha * \beta * \bar{\alpha})(t)$ and $\mathrm{H}(0, \mathrm{~s})=\alpha\left((0)_{A(0)}\right)=\alpha\left((1-s)_{J(1-s))}=\bar{\alpha}\left((s)_{J(s)}\right)=\mathrm{H}(1, \mathrm{~s}) \forall s, t \in\left(I, \widetilde{\varepsilon_{I}}\right)\right.$. Then $(\alpha * \beta * \bar{\alpha})(Q) \simeq_{c(\lambda, \delta)} \beta^{\prime}(B)$.

$(\Leftarrow)$ : Suppose $[(\alpha * \beta)(\mathrm{D}) * \bar{\alpha}(J)] \in \pi_{1}((U, \tau), c(\lambda, \delta))$.

Goal: We will show that $\pi_{1}((U, \tau), c(\lambda, \delta)) \triangle \pi_{1}((X, \tau), c(\lambda, \delta))$.

Let $\left[\beta^{\prime}(B)\right] \in \pi_{1}((U, \tau), c(\lambda, \delta))$ such that $[(\alpha * \beta)(\mathrm{D}) * \bar{\alpha}(J)]=\left[\beta^{\prime}(B)\right]$. Clearly, there exists $[o(E)]$ $\in \pi_{1}((U, \tau), c(\lambda, \delta))$ such that

$$
\begin{aligned}
{\left[\beta^{\prime}(B)\right] } & =\left[\beta^{\prime}(B)\right] \cdot[o(E)] \\
& =\left[\beta^{\prime}(B)\right] \cdot[\alpha(A) * \bar{\alpha}(J)]\left(\text { since }[o(E)] \in \pi_{1}((U, \tau), c(\lambda, \delta)) \subseteq \pi_{1}((X, \tau), c(\lambda, \delta))\right. \\
& =\left[\beta^{\prime}(B)\right] \cdot([\alpha(A)] \cdot[\bar{\alpha}(J)]) \\
& =\left[\beta^{\prime}(B)\right] \cdot([\bar{\alpha}(J)] \cdot[\alpha(A)]) \\
& =\left(\left[\beta^{\prime}(B)\right] \cdot[\bar{\alpha}(J)]\right) \cdot[\alpha(A)]
\end{aligned}
$$

then

$$
\begin{aligned}
{[(\alpha * \beta)(D) * \bar{\alpha}(J)]=\left[\beta^{\prime}(B)\right] } & =\left(\left[\beta^{\prime}(B)\right] \cdot[\bar{\alpha}(J)]\right) \cdot[\alpha(A)] \\
{[\alpha(A)] \cdot([\beta(B) * \bar{\alpha}(J)]) } & =\left(\left[\beta^{\prime}(B)\right] \cdot[\bar{\alpha}(J)]\right) \cdot[\alpha(A)] \\
{[\alpha(A)] \cdot([\beta(B)] \cdot[\bar{\alpha}(J)]) } & =\left(\left[\beta^{\prime}(B)\right] \cdot[\bar{\alpha}(J)]\right) \cdot[\alpha(A)]
\end{aligned}
$$

therefore since $\pi_{1}((U, \tau), c(\lambda, \delta))$ is a subgroup of $\pi_{1}((X, \tau), c(\lambda, \delta)) \exists[\beta(B)],\left[\beta^{\prime}(B)\right] \in \pi_{1}((X, \tau), c(\lambda, \delta)) \forall$ $[\bar{\alpha}(J)] \in \pi_{1}((U, \tau), c(\lambda, \delta))$ such that $([\beta(B)] \cdot[\bar{\alpha}(J)]),\left(\left[\beta^{\prime}(B)\right] \cdot[\bar{\alpha}(J)]\right) \in \pi_{1}((U, \tau), c(\lambda, \delta))$. Thus,

$$
[\alpha(A)]\left(\pi_{1}((U, \tau), c(\lambda, \delta))\right)=\left(\pi_{1}((U, \tau), c(\lambda, \delta))\right)[\alpha(A)] .
$$

Hence, we concludes that $\pi_{1}((U, \tau), c(\lambda, \delta)) \triangle \pi_{1}((X, \tau), c(\lambda, \delta))$.

\subsection{Necessary and sufficient condition for an element to be in the center of a fundamental group of IFTSs}

In this section, we give a necessary and sufficient condition for an element to be in a center of a fundamental group of IFTS. A necessary and sufficient condition for an element to be in a center of a group as been described in [25] that given a group $G$, the center of $G$ is define as the set of element $x \in G$ that commutes with every element of $G$ i. e.,

$$
Z(G)=\{x \in G: x y=y x \forall y \in G\} .
$$


Now, follow from the above descriptions we have the following definition:

Definition 20. The center of the fundamental group of IFTS $X$ is define as the set of all homotopy classes of loop $[\alpha(A)] \in \pi_{1}((X, \tau), c(\lambda, \delta))$ that commutes with every homotopy classes of loop $[\beta(B)] \in \pi_{1}((X, \tau), c(\lambda, \delta))$ i. e., $Z\left(\pi_{1}((X, \tau), c(\lambda, \delta))\right)=\left\{[\alpha(A)] \in \pi_{1}((X, \tau), c(\lambda, \delta)):[\alpha(A)] \cdot[\beta(B)]=[\beta(B)] \cdot[\alpha(A)] \forall[\beta(B)] \in\right.$ $\left.\pi_{1}((X, \tau), c(\lambda, \delta))\right\}$.

Theorem 8. Let $\pi_{1}((X, \tau), c(\lambda, \delta))$ be a fundamental group of IFTS $X$. Then $[\alpha(A)] \in Z\left(\pi_{1}((X, \tau), c(\lambda, \delta))\right)$ if and only if $(\alpha * o)(L) *(o * \beta)(M) \simeq_{c(\lambda, \delta)}(o * \alpha)(N) *(\beta * o)(P) \forall[\alpha(A)],[\beta(B)]$ and $[o(E)] \in \pi_{1}((X, \tau), c(\lambda, \delta))$ and for some connected IFSs $L, M, N$ and $P$ in $\left(I, \tilde{\varepsilon}_{I}\right)$.

Proof. Let $\pi_{1}((X, \tau), c(\lambda, \delta))$ be a fundamental group of IFTS $X$ and $[\alpha(A)] \in Z\left(\pi_{1}((X, \tau), c(\lambda, \delta))\right)$.

Goal: $(\alpha * o)(\mathrm{L}) *(o * \beta)(\mathrm{M}) \simeq_{c(\lambda, \delta)}(o * \alpha)(\mathrm{N}) *(\beta * o)(\mathrm{P}) \forall[\alpha(A)],[\beta(B)]$ and $[o(E)] \in \pi_{1}((X, \tau), c(\lambda, \delta))$ and for some connected IFS's L, M, N and P in $\left(I, \tilde{\varepsilon}_{I}\right)$.

To show this, it is suffices to show that there exists loop $\sigma(C)$ such that:

(i) $\sigma(\mathrm{C}) \simeq_{c(\lambda, \delta)}(\alpha * o)(\mathrm{L}) *(o * \beta)(\mathrm{M})$,

(ii) $\sigma(C) \simeq_{c(\lambda, \delta)}(o * \alpha)(\mathrm{N}) *(\beta * o)(\mathrm{P})$.

For (i): The function $H_{1}:\left(I, \widetilde{\varepsilon_{I}}\right) \times\left(I, \widetilde{\varepsilon_{I}}\right) \rightarrow(X, \tau)$ defined by

$$
H_{1}(t, s)= \begin{cases}\alpha\left(((t)(s)+(2 t)(1-s))_{A((t)(s)+(2 t)(1-s))),}\right. & \text { if } 0 \leq t \leq \frac{1+s}{2} \\ c(\lambda, \delta)=o\left(t_{E(t)}\right), & \text { if } \frac{1+s}{2} \leq t \leq 1\end{cases}
$$

is a homotopy between $\alpha(A)$ and $(\alpha * o)(\mathrm{L})$ i. e., $H_{1}$ is continuous by Lemma 2,

$$
\begin{aligned}
H_{1}(t, 0) & = \begin{cases}\alpha\left((2 t)_{A(2 t)}\right), & \text { if } 0 \leq t \leq \frac{1+s}{2} \\
c(\lambda, \delta)=o\left(t_{E(t)}\right), & \text { if } \frac{1+s}{2} \leq t \leq 1\end{cases} \\
& =(\alpha * o)\left(t_{L(t)}\right),
\end{aligned}
$$

$H_{1}(\mathrm{t}, 1)=\alpha\left(t_{A(t)}\right)$ and $H_{1}(0, \mathrm{~s})=\alpha\left(0_{A(0)}\right)=c(\lambda, \delta)=o\left(1_{E(1)}\right)=H_{1}(1, \mathrm{~s})$. Then $\alpha(A) \simeq_{c(\lambda, \delta)}(\alpha * o)(\mathrm{L})$. Also, the function $H_{2}:\left(I, \widetilde{\varepsilon_{I}}\right) \times\left(I, \widetilde{\varepsilon_{I}}\right) \rightarrow(X, \tau)$ defined by

$$
H_{2}(t, s)= \begin{cases}c(\lambda, \delta)=o\left(t_{E(t)}\right), & \text { if } 0 \leq t \leq \frac{1-s}{2} \\ \beta\left(((t)(s)+(2 t-1)(1-s))_{B((t)(s)+(2 t-1)(1-s))}\right), & \text { if } \frac{1-s}{2} \leq t \leq 1\end{cases}
$$

is a homotopy between $\beta(B)$ and $(o * \beta)(\mathrm{M})$ i. e., $\mathrm{H}_{2}$ is continuous by Lemma 2 ,

$$
\begin{aligned}
H_{2}(t, 0) & = \begin{cases}c(\lambda, \delta)=o\left(t_{E(t)}\right), & \text { if } 0 \leq t \leq \frac{1-s}{2} \\
\beta\left((2 t-1)_{B(2 t-1)}\right), & \text { if } \frac{1-s}{2} \leq t \leq 1\end{cases} \\
& =(o * \beta)\left(\left(t_{M(t)}\right)\right),
\end{aligned}
$$

$H_{2}(\mathrm{t}, 1)=\beta\left(t_{B(t)}\right)$ and $H_{2}(0, \mathrm{~s})=c(\lambda, \delta)=o\left(0_{E(0)}\right)=\beta\left(1_{B(1)}\right)=H_{2}(1, \mathrm{~s})$, then $\beta(B) \simeq_{c(\lambda, \delta)}(o * \beta)(\mathrm{M})$. Thus, the function $\mathrm{H}:\left(I, \widetilde{\varepsilon_{I}}\right) \times\left(I, \widetilde{\varepsilon_{I}}\right) \rightarrow(X, \tau)$ defined by $\mathrm{H}(\mathrm{t}, \mathrm{s})=H_{1}(\mathrm{t}, \mathrm{s}) * H_{2}(\mathrm{t}, \mathrm{s})$ is a homotopy between $\sigma$ and $(\alpha * o)(\mathrm{L}) *(o * \beta)(\mathrm{M}) i$. e., $\mathrm{H}$ is the composition of two continuous functions, $\mathrm{H}(\mathrm{t}, 0)=(\alpha * o)(\mathrm{L}) *(o * \beta)(\mathrm{M}), \mathrm{H}(\mathrm{t}$, $1)=(\alpha * \beta)(\mathrm{F})=\sigma(\mathrm{C})$ and $\mathrm{H}(0, \mathrm{~s})=H_{1}(0, \mathrm{~s}) * H_{2}(0, \mathrm{~s})=H_{1}(1, \mathrm{~s}) * H_{2}(1, \mathrm{~s})=\mathrm{H}(1, \mathrm{~s})$. Therefore $\sigma(C) \simeq_{c(\lambda, \delta)}(\alpha * o)(\mathrm{L})$ $*(o * \beta)(\mathrm{M})$.

For (ii): $K_{1}:\left(I, \widetilde{\varepsilon_{I}}\right) \times\left(I, \widetilde{\varepsilon_{I}}\right) \rightarrow(X, \tau)$ defined by

$$
K_{1}(t, s)= \begin{cases}c(\lambda, \delta)=o\left(t_{E(t)}\right), & \text { if } 0 \leq t \leq \frac{1-s}{2} \\ \alpha\left(((t)(s)+(2 t-1)(1-s))_{A((t)(s)+(2 t-1)(1-s))}\right), & \text { if } \frac{1-s}{2} \leq t \leq 1\end{cases}
$$


is a homotopy between $\alpha(A)$ and $(o * \alpha)(\mathrm{N})$ i. e., $K_{1}$ is continuous by Lemma 2 ,

$$
\begin{aligned}
K_{1}(t, 0) & = \begin{cases}c(\lambda, \delta)=o\left(t_{E(t)}\right), & \text { if } 0 \leq t \leq \frac{1-s}{2} \\
\alpha\left((2 t-1)_{A(2 t-1)}\right), & \text { if } \frac{1-s}{2} \leq t \leq 1\end{cases} \\
& =(o * \beta)\left(\left(t_{M(t)}\right)\right),
\end{aligned}
$$

$K_{1}(\mathrm{t}, 1)=\alpha\left(t_{A(t)}\right)$ and $K_{1}(0, \mathrm{~s})=c(\lambda, \delta)=o\left(0_{E(0)}\right)=o\left(1_{E(1)}\right)=K_{1}(1, \mathrm{~s})$, then $\alpha(A) \simeq_{c(\lambda, \delta)}(o * \alpha)(\mathrm{N})$. Also the function $K_{2}:\left(I, \widetilde{\varepsilon_{I}}\right) \times\left(I, \widetilde{\varepsilon_{I}}\right) \rightarrow(X, \tau)$ defined by

$$
K_{2}(t, s)= \begin{cases}\beta\left(((t)(s)+(2 t)(1-s))_{B((t)(s)+(2 t)(1-s))),}\right. & \text { if } 0 \leq t \leq \frac{1+s}{2} \\ c(\lambda, \delta)=o\left(t_{E(t)}\right), & \text { if } \frac{1+s}{2} \leq t \leq 1\end{cases}
$$

is a homotopy between $\beta(B)$ and $(\beta * o)(P)$ i. e., $K_{2}$ is continuous by Lemma 2,

$$
\begin{aligned}
K_{2}(t, 0) & = \begin{cases}\beta\left((2 t)_{B(2 t)}\right), & \text { if } 0 \leq t \leq \frac{1+s}{2} \\
c(\lambda, \delta)=o\left(t_{E(t)}\right), & \text { if } \frac{1+s}{2} \leq t \leq 1\end{cases} \\
& =(\beta * o)\left(t_{P(t)}\right),
\end{aligned}
$$

$K_{2}(\mathrm{t}, 1)=\beta\left(t_{B(t)}\right)$ and $K_{2}(0, \mathrm{~s})=\beta\left(0_{B(0)}\right)=c(\lambda, \delta)=o\left(1_{E(1)}\right)=K_{2}(1, \mathrm{~s})$. Then $\alpha(A) \simeq_{c(\lambda, \delta)}(\beta * o)(\mathrm{P})$.

Hence the function $\mathrm{K}::\left(I, \widetilde{\varepsilon_{I}}\right) \times\left(I, \widetilde{\varepsilon_{I}}\right) \rightarrow(X, \tau)$ defined by $\mathrm{K}(\mathrm{t}, \mathrm{s})=K_{1}(\mathrm{t}, \mathrm{s}) * K_{2}(\mathrm{t}, \mathrm{s})$ is a homotopy between $\sigma(C)$ and $(o * \alpha)(\mathrm{N}) *(\beta * o)(\mathrm{P}) i$. e., $\mathrm{K}$ is the composition of two continuous functions, $\mathrm{K}(\mathrm{t}, 0)=$ $\left(\varepsilon_{p} * \mathrm{f}\right) *\left(\mathrm{f} * \varepsilon_{p}\right)(\mathrm{t}), \mathrm{K}(\mathrm{t}, 1)=(\mathrm{f} * \mathrm{~g})(\mathrm{t})=\sigma(\mathrm{t})$ and $\mathrm{K}(0, \mathrm{t})=K_{1}(0, \mathrm{~s}) * K_{2}(0, \mathrm{~s})=K_{1}(1, \mathrm{~s}) * K_{2}(1, \mathrm{~s})=\mathrm{K}(1, \mathrm{x})$. Then $\sigma(C) \simeq_{c(\lambda, \delta)}(o * \alpha)(\mathrm{N}) *(\beta * o)(\mathrm{P})$. Thus, the result follows. $\left(I, \tilde{\varepsilon}_{I}\right)$,

$(\Leftarrow)$ Suppose $(\alpha * o)(\mathrm{L}) *(o * \beta)(\mathrm{M}) \simeq_{c(\lambda, \delta)}(o * \alpha)(\mathrm{N}) *(\beta * o)(\mathrm{P})$ for some connected IFSs L, M, N and P in

Goal: $[\alpha(A)] \in Z\left(\pi_{1}((X, \tau), c(\lambda, \delta))\right)$.

Since $(\alpha * \beta)(\mathrm{F})=(\alpha * o)(\mathrm{L}) *(o * \beta)(\mathrm{M}) \simeq_{c(\lambda, \delta)}(o * \alpha)(\mathrm{N}) *(\beta * o)(\mathrm{P})=(\beta * \alpha)(\mathrm{H})$ for some connected IFSs $\mathrm{L}, \mathrm{M}, \mathrm{N}$ and $\mathrm{P}$ in $\left(I, \tilde{\varepsilon}_{I}\right)$.

Then $(\alpha * \beta)(\mathrm{F}) \simeq_{c(\lambda, \delta)}(\beta * \alpha)(\mathrm{H})$ which implies $[(\alpha * \beta)(\mathrm{F})]=[(\beta * \alpha)(\mathrm{H})]$ and by the concatenation of loop defined on $\pi_{1}((X, \tau), c(\lambda, \delta))$, we have $[\alpha(A)] \cdot[\beta(B)]=[\beta(B)] \cdot[\alpha(A)]$. Thus, the result follows.

\subsection{Quotient Fundamental Group of IFTSs}

In this section, we deduced the quotient group under fundamental group of IFTS from general description of quotient group. As it is generally described in [25] that given a group $G$ and a normal subgroup $N$, a set $G / N$ define by

$$
G / N=\{g N: g \in G\}
$$

is called a quotient group.

Definition 21. Let $\pi_{1}((X, \tau), c(\lambda, \delta))$ be fundamental group of IFTS and $\pi_{1}((U, \tau), c(\lambda, \delta))$ be its normal subgroup, a set $\left(\pi_{1}((X, \tau), c(\lambda, \delta))\right) /\left(\pi_{1}((U, \tau), c(\lambda, \delta))\right)$ define by

$$
\left(\pi_{1}((X, \tau), c(\lambda, \delta))\right) /\left(\pi_{1}((U, \tau), c(\lambda, \delta))\right)=\left\{[\alpha(A)]\left(\pi_{1}((U, \tau), c(\lambda, \delta))\right):[\alpha(A)] \in \pi_{1}((X, \tau), c(\lambda, \delta))\right\}
$$

is called a quotient group.

Theorem 9. Let $\pi_{1}((X, \tau), c(\lambda, \delta))$ be fundamental group of IFTS and $Z\left(\pi_{1}((X, \tau), c(\lambda, \delta))\right)$ be center of $\pi_{1}((X, \tau), c(\lambda, \delta))$. Then the set $\pi_{1}((X, \tau), c(\lambda, \delta)) / Z\left(\pi_{1}((X, \tau), c(\lambda, \delta))\right)$ is a quotient group.

Proof. Clearly, $Z\left(\pi_{1}((X, \tau), c(\lambda, \delta))\right)$ subgroup of $\pi_{1}((X, \tau), c(\lambda, \delta))$. We only need to show that $\mathrm{Z}\left(\pi_{1}((X, \tau), c(\lambda, \delta))\right)$ is normal. 
Let $[o(E)] \in \pi_{1}((X, \tau), c(\lambda, \delta))$, then $\exists \beta(B) \in Z\left(\pi_{1}((X, \tau), c(\lambda, \delta))\right)$ such that $[o(E)] \cdot[\beta(B)] \in$ $Z\left(\pi_{1}((X, \tau), c(\lambda, \delta))\right)$ for some connected IFSs A, B and C in $\left(I, \tilde{\varepsilon}_{I}\right)$

$$
\begin{aligned}
{[o(E)] \cdot[\beta(B)]=} & {[\alpha(A) * \bar{\alpha}(J)] \cdot[\beta(B)] } \\
& =([\alpha(A)] \cdot[\bar{\alpha}(J)]) \cdot[\beta(B)] \\
& =[\alpha(A)] \cdot([\bar{\alpha}(J)] \cdot[\beta(B)]) \\
& =[\alpha(A)] \cdot([\beta(B)] \cdot[\bar{\alpha}(J)]) \\
\Rightarrow[\beta(B)] & =[\alpha(A)] \cdot([\beta(B)] \cdot[\bar{\alpha}(J)]) \\
& =([\alpha(A)] \cdot[\beta(B)]) \cdot[\bar{\alpha}(J)] \\
& =[(\alpha * \beta)(F) * \bar{\alpha}(J)] .
\end{aligned}
$$

Then $Z\left(\pi_{1}((X, \tau), c(\lambda, \delta))\right)$ is normal. Therefore we concludes that the set $\pi_{1}((X, \tau), c(\lambda, \delta)) / Z\left(\pi_{1}((X, \tau), c(\lambda, \delta))\right)$ is a quotient group.

\section{Description of centralizer of the fundamental group of IFTSs}

In this section, we deduced the centralizer of an element in a fundamental group of IFTS from general description of centralizer of an element in a group. As it is generally described that given a group $G$, the centralizer of an element in $x \in G$ is define as a set of elements $G$ that commutes with every element of $x$ i. e.,

$$
C(x)=\{y \in G: x y=y x \forall x \in G\} .
$$

Further applications includes; centralizers of nilpotent group elements in semisimple algebraic groups [27]; the isomorphism type of the centralizer of an element in a lie group [28]; centralizers of semisimple elements in the finite classical groups [29].

Now, we have the following definition:

Definition 22. Let $\pi_{1}((X, \tau), c(\lambda, \delta))$ be a fundamental group of IFTS $X$ and $[\alpha(A)]$ be element of $\pi_{1}((X, \tau), c(\lambda, \delta))$. We define the centralizer of $[\alpha(A)]$ to be the set of all homotopy classes of loop $[\beta(B)] \in$ $\pi_{1}((X, \tau), c(\lambda, \delta))$ that commutes with $[\alpha(A)]$.

$$
C([\alpha(A)])=\left\{[\beta(B)] \in \pi_{1}((X, \tau), c(\lambda, \delta)):[\alpha(A)] \cdot[\beta(B)]=[\beta(B)] \cdot[\alpha(A)]\right\} .
$$

Theorem 10. Let $\pi_{1}((X, \tau), c(\lambda, \delta))$ be a fundamental group of IFTS $X$. Then $C([\alpha(A)])$ is a centralizer of $[\alpha(A)] \in$ $\pi_{1}((X, \tau), c(\lambda, \delta))$ if and only if $(\alpha * o)(F) *(o * \beta)(G) \simeq_{c(\lambda, \delta)}(o * \alpha)(H) *(\beta * o)(J) \forall[\beta(B)]$ and identity $[o(E)]$ $\in \pi_{1}((X, \tau), c(\lambda, \delta))$ and for some connected IFSs $F, G, H$ and $J$ in $\left(I, \tilde{\varepsilon}_{I}\right)$.

Acknowledgments: The author wish to express his profound gratitude to the reviewers for their useful remarks.

Conflicts of Interest: "The author declare no conflict of interest."

\section{References}

[1] Atanassov, K. T. (1983). Intuitionistic fuzzy sets VII ITKRs Session. Sofia, June, 1, 1983.

[2] Zadeh, L. A. (1965). Fuzzy sets. Information and control, 8(3), 338-353.

[3] Az-Zo?bi, E. A., Marashdeh, M. F., \& Uzbashy, R. F. (2014). The fundamental group of intuitionistic fuzzy topological spaces. Applied Mathematical Sciences, 8(157), 7829-7843.

[4] Osmanoglu, I., \& Tokat, D. (2013). On intuitionistic fuzzy soft topology. General Mathematics Notes, 19(2), 59-70.

[5] Babitha, K. V., \& Sunil, J. J. (2011). Generalized intuitionistic fuzzy soft sets and its applications. General Mathematics Notes, 7(2), 2219-7184.

[6] Saroja, R., \& Kalaichelvi, A. (2015). Intuitionistic fuzzy neutrosophic soft topological spaces. International Journal of Innovative Research in Science, Engineering and Technology, 4(5), 3338-3345.

[7] Deschrijver, G., Cornelis, C., \& Kerre, E. E. (2004). On the representation of intuitionistic fuzzy t-norms and t-conorms. IEEE Transactions on Fuzzy Systems, 12(1), 45-61.

[8] Deschrijver, G., Cornelis, C., \& Kerre, E. (2002). Intuitionistic fuzzy connectives revisited. In 9th International Conference on Information Processing and Management of Uncertainty in Knowledge-Based Systems (IPMU 2002) (pp. 1839-1844). 
[9] Liu, H. W., \& Wang, G. J. (2007). Multi-criteria decision-making methods based on intuitionistic fuzzy sets. European Journal of Operational Research, 179(1), 220-233.

[10] Ersoy, B. A., Onar, S., Hila, K., \& Davvaz, B. (2013). Some properties of intuitionistic fuzzy soft rings. Journal of Mathematics, 2013, Article ID 650480

[11] Jiang, D., \& Wang, Y. (2018). A new entropy and its properties based on the improved axiomatic definition of intuitionistic fuzzy entropy. Mathematical Problems in Engineering, 2018, Article ID 7606801.

[12] Mondal, S. P., Goswami, A., \& Kumar De, S. (2019). Nonlinear triangular intuitionistic fuzzy number and its application in linear integral equation. Advances in Fuzzy Systems, 2019, Article ID 4142382.

[13] Li, C., \& Jin, J. (2018). A scalar expected value of intuitionistic fuzzy random individuals and its application to risk evaluation in insurance companies, Maths. Problems in Enginearing, 2018, Article ID 8319859

[14] Güner, E., \& Balci, S. (2007). On the fuzzy sheaf of the fundamental groups. Taiwanese Journal of Mathematics, 1057-1062.

[15] Girija, S., \& Ilango, G. (2014). Some more results on intuitionistic semi open sets. International Journal of Engineering Research and Applications, 4(11), 70-74.

[16] Al-Qubati, A. A., \& Al-qahtani, H. F. (2015). On b-separation axioms in intuistionistic Fuzzy Topological Spaces. International Journal of Mathematics Trends and Technology, 21, 83-93.

[17] Toker, D. (1997). An introduction to intuitionistic fuzzy topological spaces. Fuzzy Sets and Systems, 88(1), 81-89.

[18] Pinter, C.C. (1990). A book of abstract algebra, Dover Publications, Inc., Mineola, New York.

[19] Klüver, H. (2008). The unitary character group of abelian unipotent groups. Munster Journal of Mathematics, 1, 181-220.

[20] Arveson, W. (1966). A theorem on the action of abelian unitary groups. Pacific Journal of Mathematics, 16(2), $205-212$.

[21] Clark, W.E. (2001). Elementary abstract algebra. University of South Floria.

[22] Dye, R. H. (1984). Maximal subgroups of symplectic groups stabilizing spreads. Journal of Algebra, 87(2), 493-509.

[23] Li, X., Li, R.H. and Wang, L. (2008). 3-permutable aubgroups and p-nilpotency of finite group II, Isreal Journal of Mathematics, 164 75-85.

[24] Asaad, M., \& Abou-Zaid, S. (1993). Fuzzy subgroups of nilpotent groups. Fuzzy Sets and Systems, 60(3), $321-323$.

[25] Milne, J.S. (2011). A textbook for algebraic groups, lie groups, and their arithmetic subgroups version 3.00, 2011, Available at www.jmilne.org/math/.

[26] Zeng, L. (2011). Two theorems about nilpotent subgroup. Applied Mathematics, 2(05), 562-564.

[27] Elkington, G. B. (1972). Centralizers of unipotent elements in semisimple algebraic groups. Journal of Algebra, 23(1), 137-163.

[28] Duan, H., \& Liu, S. (2013). The isomorphism type of the centralizer of an element in a Lie group. Journal of Algebra, $376,25-45$.

[29] Carter, R. W. (1981). Centralizers of semisimple elements in the finite classical groups. Proceedings of the London Mathematical Society, 3(1), 1-41.

(C) 2020 by the author; licensee PSRP, Lahore, Pakistan. This article is an open access article distributed under the terms and conditions of the Creative Commons Attribution (CC-BY) license (http://creativecommons.org/licenses/by/4.0/). 